\title{
A congenital direct Gerbode defect in a 2-year-old girl
}

\author{
Du Bowen ${ }^{1}$, Kai Bai ${ }^{1}$, Peng fei $\mathrm{Wu}^{1}$, Ya Nan $\mathrm{Lu}^{1}$, and Kun Sun ${ }^{1}$ \\ ${ }^{1}$ Xinhua Hospital Affiliated to Shanghai Jiaotong University School of Medicine
}

July 28, 2020

\begin{abstract}
The Gerbode defect is a special type of ventricle septum defect causing a communication between the left ventricle and right atrium. In this article, we present a rare case of 2-year-old congenital Gerbode defect. The location of this defect was special, just at the interventricular septum between the tricuspid and mitral valve. She underwent the closure of the defect with an autologous pericardial patch in station of mild hypothermic cardiopulmonary bypass.
\end{abstract}

\section{CASE PRESENTATION}

A 2-year-old girl was referred to our hospital because of cardiac murmurs found in 1 month after birth. She had sweating,poor appetite and a history of thalassemia with her mother.There were no other symptoms except $2 / 6$ rough systolic murmurs were found between left parasternal 3-4 ribs. The ECG showed $\mathrm{I}^{\circ}$ atrial ventricular block(Figure 1A). Chest radiograph showed enlarged heart and lung coarse texture(Figure 1B). Echocardiogram showed a patent foramen ovale(PFO) and an $11.2 \mathrm{~mm}$ ventricular septal defect(VSD), at the upper membranous septum between the tricuspic and mitrial valve, causing a $3.1 \mathrm{~mm}$ high-velocity systolic flow shunt $(4 \mathrm{~m} / \mathrm{s})$ from left ventricle $(\mathrm{LV})$ to the right atrium(RA) and the flow persisted to the diastole (Figure 2A and 2B). The pulmonary artery was a little wider and the pressure was normal according to a faint pulmonary insufficiency $(1.42 \mathrm{~m} / \mathrm{s})$. But we didn't find the dilation of right ventricle or other chambers even if the defect was so large for her. She underwent the closure of the VSD with an autologous pericardial patch and surgical suture of PFO using mild hypothermic cardiopulmonary bypass(CPB). Intraoperatively, the PFO was $3 \mathrm{mmx} 3 \mathrm{~mm}$ and the VSD was $10 \mathrm{~mm} \times 8 \mathrm{~mm}$ above the septal leaflets of the tricuspid valve(TV) but below the mitral valve(MV)that caused a left ventricular-to-right atrial communication(Figure $2 \mathrm{C}$ and $2 \mathrm{D}$ ). Her postoperative course was uneventful,and in the follow-up after 3 months, postoperative echocardiography also revealed no residual shunt.

\section{DISCUSSION}

The Gerbode defect is a rare communication between the heart chambers, which connects the left ventrical to the right atrium.It was named after Gerbode $^{1}$ successfully performed the operation in five patients in 1958.Initially,the author illustrated that the lesion consisted of a high ventricular septal defect associated with a defect of the septal leaflet of the tricuspid valve which allowed left ventricular blood to enter the right atrium. But gradually this special type of VSD causing a LV-to-RA shunt is all called Gerbode defect whether it accompany with the defect of the septal leaflet of the tricuspid valve or not ${ }^{2}$. The cause of the defect can be congenital or aquired.Aquired defect is often a complication of endocarditis ${ }^{3}$, myocardial infarction ${ }^{4}$, blunt chest trauma ${ }^{5}$ or cardiac surgery ${ }^{6}$. Acquired LV-RA communication is increasingly being reported. The LV-to RA shunt accounts for only $0.08 \%$ of intracardiac shunts ${ }^{7}$ and $<1 \%$ of all congenital cardiac defects ${ }^{8}$. Congenital Gerbode defect is so rare that researchers observed only six cases at the Children's Memorial Hospital in Chicago between 1990 and $2008^{2}$. Riemenschneider and Moss ${ }^{9}$ have described two types of LVRA communication based on whether the communication is direct or indirect(though the tricuspic valve). According to the location of the defect, Sakakibara and Konno ${ }^{10}$ classified it into 3 types. Type 1,also called 
the direct type without tricuspic regurgitation, is a supravavular defect occuring in the atrialventricular septum above TV.Type 2 is a infravavular defect occuring in the memberous, muscular intraventricular septum or endocardial cushion below the TV.The shunt of this type defect was often confused with the tricuspic regurgitation,so it was called the indirect type.Type 3 is an intermediate defect with both supravalvular and infravalvular components. The type 2 and 3 may have many variants to septal leaflet anomalies of the TV, including a cleft, widened commissural space, perforation, abnormal chordae and other deformities. Type 1 is common in acquired cases but congenital type is very rare. This case was a congenital type 1 defect $^{2,11}$.

Echocardiography is the most useful diagnosis method and is noninvasive and radiation-free. In ordinary VSD, we could usually find the blood flow goes directly into the right ventricle during the systole but doesn't last until diastole and the pulmonary artery congestion and right ventricle enlargement is usually happen before the right atrium enlargement. Silbiger ${ }^{12}$ et al discribed clues suggesting the presence of a Gerbode shunt include (1) atypical jet direction, (2) persistent shunt flow into diastole, (3) the absence of ventricular septal flattening, (4) the absence of right ventricular hypertrophy, and (5) the presence of a normal diastolic pulmonary artery pressure estimated from the pulmonic regurgitant velocity. Cardiac magnetic resonance (CMR) is one of the adjuvant techniques to reveal further detailed anatomical and physiological information, but it is expensive and uneasy to obtain for children . Cardiac catheterization is invasive, so it is not necessary for diagnosis unless to evaluate the hemodynamics and pressure.

Some researchers recommend that the treatment of this defect is according to the severity of symptoms which is associated with the size, location, magnitude of shunt, flow volume, development time magnitude of shunt. Asymptomatic, without circulatory overload or small defects with insignificant intracardiac shunt could be managed conservatively by following up rather than surgery ${ }^{2}$. But in our opinion, continuous shunt not only increase the load of the heart, but also increase the risk of infective endocarditis and the surgery is safe, so we advocate to treat it no matter whether the symptom is significant or not. The treatment includes the transcatheter closure and the patch closure surgery. The percutaneous transcatheter closure techniques has been used mostly in acquired cases ${ }^{11,13}$ or high-risk surgical candidates due to previous valve replacement, advanced age, anticoagulation, and multiple comorbidities ${ }^{14}$. The LV-to-RA communication was corrected by surgery traditionally and surgical closure has been demonstrated to be with excellent outcome and recommended for closure of all direct defects ${ }^{2}$. In this case,the little girl had some symptoms of right ventricle overload including sweating and poor appetite. The size of the lesion $(11.3 \mathrm{~mm})$ and the magnitude of shunt was large for 2-year-old girl.Thus, we chose the patch to close the lesion.

\section{ACKNOWLEDGEMENTS}

The authors thank Chen Sun, Wu Yurong and Sun Kun.

Conflict of interest : none declared.

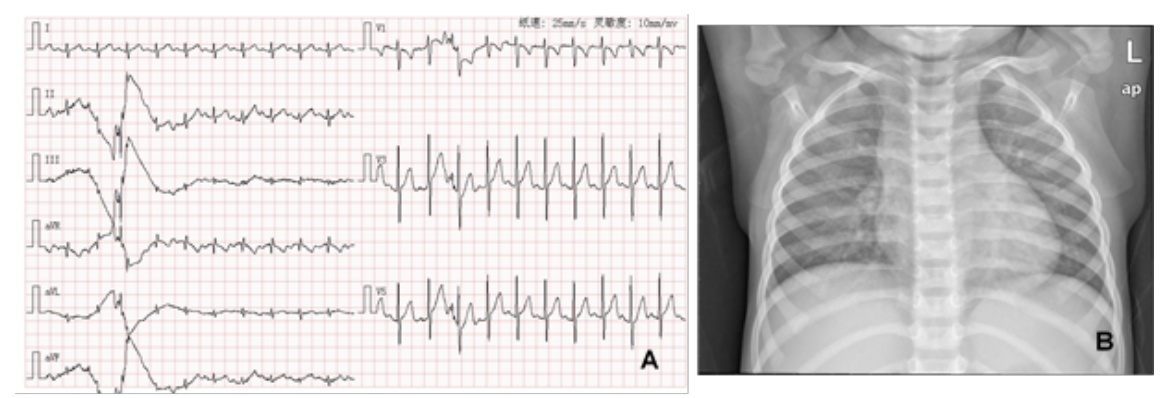

Figure 1 :(A )The ECG of this patient showed I atrial ventricular block,(B )The chest radiograph of this patient showed enlarged heart and lung coarse texture. 

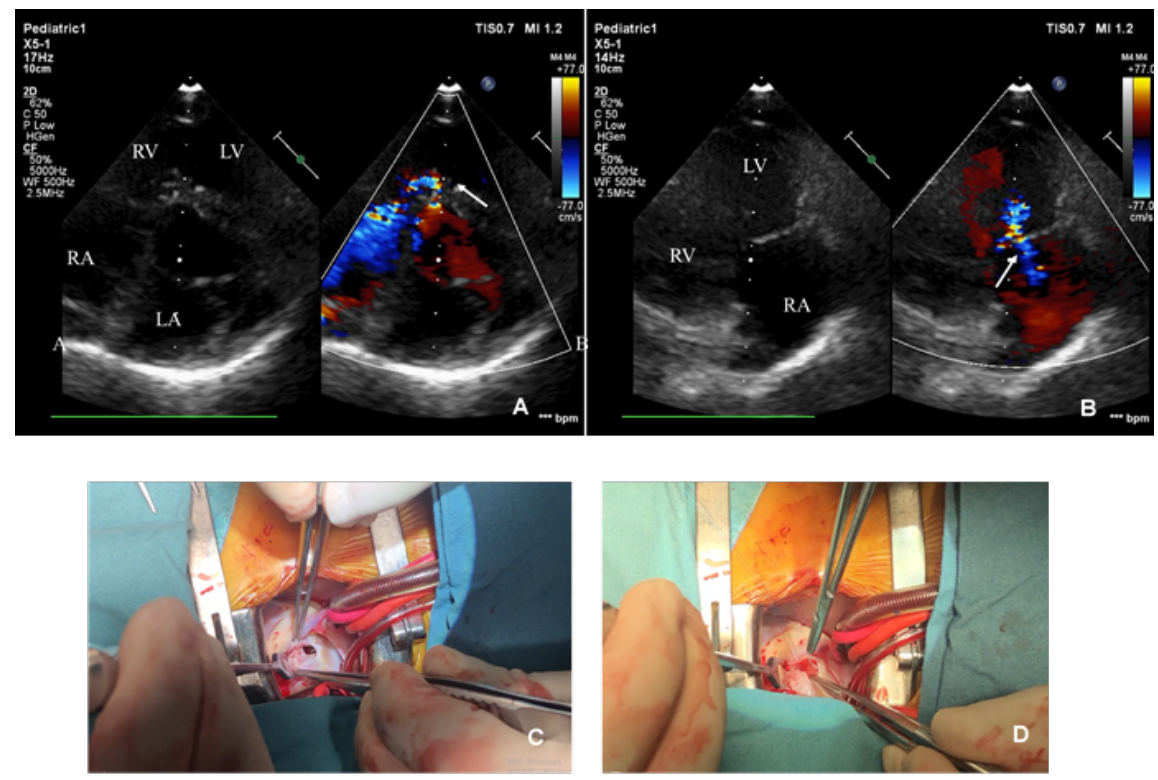

Figure 2 :(A ) In the short-axis view of the great vessels view with color Doppler demonstrated the left ventricular to right atrial shunt and the defect was just blow the MV but above the TV.The blue shunt is often confused with tricuspid regurgitation.(The white arrow shows the LV-to-RA shunt),(B )In parasternal long-axis right ventricular inflow tract view with color Doppler demonstrated the left ventricular to right atrial shunt. (The white arrow shows the LV-to-RA shunt),(C)The VSD in operation in right atrial view,(D )The VSD was closed with a continuous technique using 6-0 prolene suture with an autologous pericardial patch.

\section{REFERENCES}

1. Gerbode F, Hultgren H, Melrose D, Osborn J. Syndrome of left ventricular-right atrial shunt; successful surgical repair of defect in five cases, with observation of bradycardia on closure. Annals of surgery. 1958;148(3):433-446.

2. Kelle AM, Young L, Kaushal S, Duffy CE, Anderson RH, Backer CL. The Gerbode defect: the significance of a left ventricular to right atrial shunt. Cardiology in the young.2009;19 Suppl 2:96-99.

3. Battin M, Fong LV, Monro JL. Gerbode ventricular septal defect following endocarditis. European journal of cardio-thoracic surgery : official journal of the European Association for Cardio-thoracic Surgery. 1991;5(11):613-614.

4. Newman JN, Jr., Rozanski L, Kreulen T. Acquired left ventricular to right atrial intracardiac shunt after myocardial infarction: a case report and review of the literature. Journal of the American Society of Echocardiography : official publication of the American Society of Echocardiography.1996;9(5):716-720.

5. Olsovsky MR, Topaz O, DiSciascio G, Vetrovec GW. Acute traumatic ventricular septal rupture. American heart journal. 1996;131(5):1039-1041.

6. Amirghofran AA, Emaminia A. Left ventricular-right atrial communication (Gerbode-type defect) following mitral valve replacement. Journal of cardiac surgery.2009;24(4):474-476.

7. Taskesen T, Prouse AF, Goldberg SL, Gill EA. Gerbode defect: Another nail for the 3D transesophagel echo hammer? The international journal of cardiovascular imaging.2015;31(4):753-764.

8. Tidake A, Gangurde P, Mahajan A. Gerbode Defect-A Rare Defect of Atrioventricular Septum and Tricuspid Valve. Journal of clinical and diagnostic research : JCDR.2015;9(9):Od06-08. 
9. Riemenschneider TA, Moss AJ. Left ventricular-right atrial communication. The American journal of cardiology. 1967;19(5):710-718.

10. Sakakibara S, Konno S. Congenital aneurysm of the sinus of Valsalva. Anatomy and classification.American heart journal. 1962;63:405-424.

11. Yuan SM. A Systematic Review of Acquired Left Ventricle to Right Atrium Shunts (Gerbode Defects).Hellenic journal of cardiology $:$ HJC $=$ Hellenike kardiologike epitheorese. 2015;56(5):357-372.

12. Silbiger JJ, Kamran M, Handwerker S, Kumar N, Marcali M. The Gerbode defect: left ventricular to right atrial communication-anatomic, hemodynamic, and echocardiographic features. Echocardiography (Mount Kisco, NY). 2009;26(8):993-998.

13. Cabalka AK, Hagler DJ, Mookadam F, Chandrasekaran K, Wright RS. Percutaneous closure of left ventricular-to-right atrial fistula after prosthetic mitral valve rereplacement using the Amplatzer duct occluder. Catheterization and cardiovascular interventions : official journal of the Society for Cardiac Angiography \& Interventions. 2005;64(4):522-527.

14. Prifti E, Ademaj F, Baboci A, Demiraj A. Acquired Gerbode defect following endocarditis of the tricuspid valve: a case report and literature review. Journal of cardiothoracic surgery. 2015;10:115.
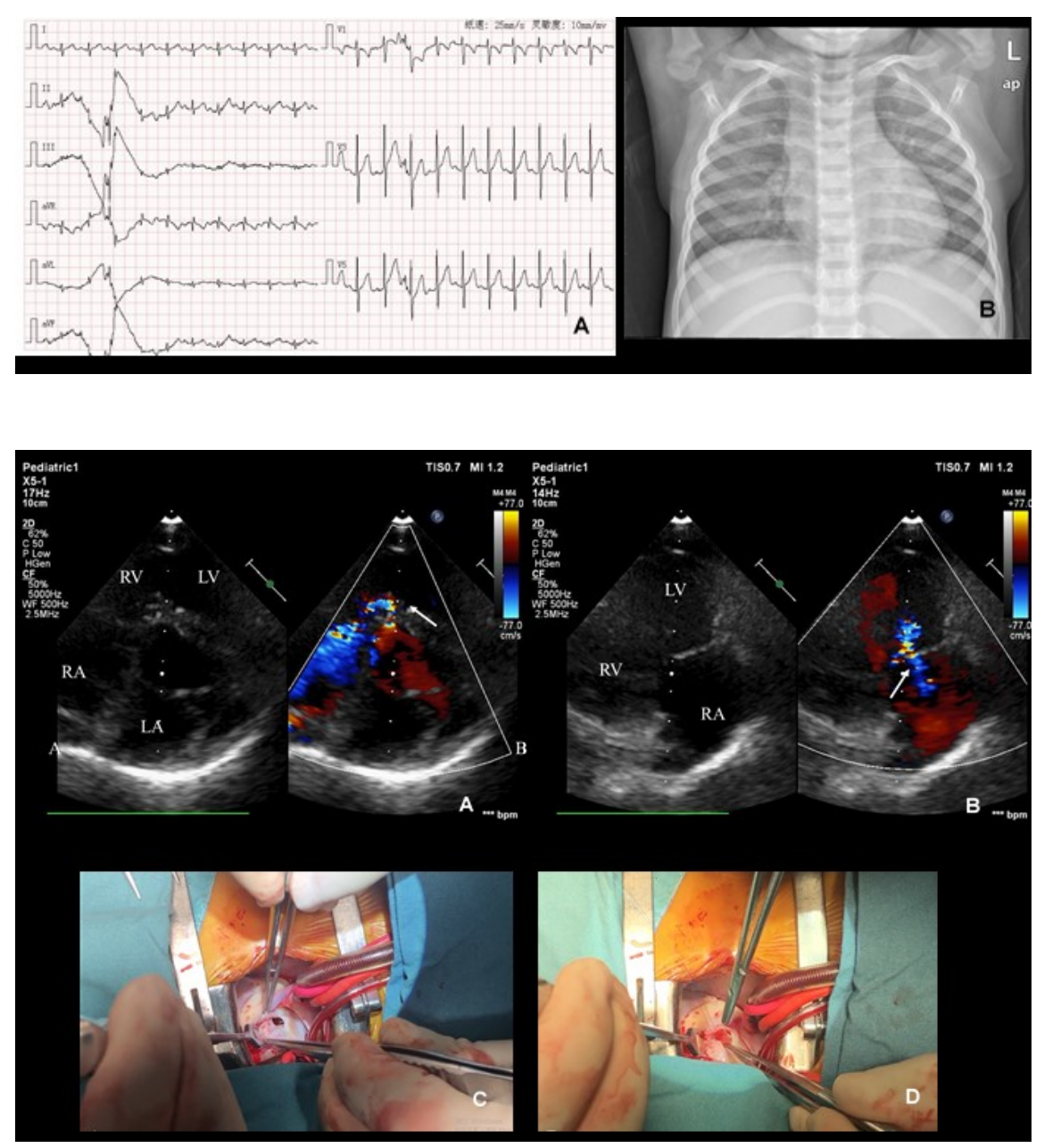